\title{
La praxis interseccional en las políticas públicas: el poder de las metáforas
}

\section{Intersectional praxis in policies: the power of metaphors}

\author{
Jone Martínez-Palacios \\ Universidad del País Vasco / Euskal Herriko Unibertsitatea (España) \\ ORCID: https://orcid.org/0000-0002-9354-3148 \\ jone.martinez@ehu.eus
}

\section{NOTA BIOGRÁFICA}

Jone Martínez-Palacios es profesora titular en el Departamento de Ciencia Política y de la Administración de la Universidad del País Vasco/Euskal Herriko Unibertsitatea. Miembro del Grupo de Investigación PARTE HARTUZ. Doctora en Ciencia Política y de la Administración.

Andere Ormazabal Gaston

Universidad del País Vasco / Euskal Herriko Unibertsitatea (España)

ORCID: https://orcid.org/0000-0002-6886-5137

andere.ormazabal@ehu.eus

\section{NOTA BIOGRÁFICA}

Andere Ormazabal es profesora en el Departamento de Ciencia Política y de la Administración de la Universidad del País Vasco/Euskal Herriko Unibertsitatea. Miembro del Grupo de Investigación Consolidado PARTE HARTUZ (UFI EMAN). Doctora en Ciencia Política y de la Administración.

\section{RESUMEN}

En contextos políticos y administrativos en los que se han instalado las prácticas de la nueva gestión pública mediante una visión neoempresarial, las políticas públicas se orientan a través de una noción de la eficiencia que pasa por organizar los recursos públicos de una forma que garantice la acumulación de capital en sus distintas expresiones. Frente a esta tendencia la teoría crítica feminista propone estructurar la acción pública de una forma no binaria. Este texto explora las posibilidades de la herramienta de la interseccionalidad para ese fin. Concretamente, indaga en la potencialidad del pensamiento metafórico a partir de la metáfora de los cuerpos marcados en el diseño, implementación y evaluación de las políticas públicas.

\section{PALABRAS CLAVE}

Teoría crítica feminista; Interseccionalidad; Profundización democrática; Cuerpos marcados; Acción pública.

\begin{abstract}
In political and administrative contexts in which the practices of the new public management have been installed, public policies are oriented towards efficiency, in sense of organizing public resources to the accumulation of capital in any of his forma. Faced with this reality, Feminist Critical Theory proposes to structure public action in non-binary form. This text explores the possibilities of the tool of intersectionality
\end{abstract}


for that purpose. To this end, the metaphoric thought and the metaphor of marked bodies is presented as potentially useful in the design, implementation and evaluation of public policies.

\section{KEYWORDS}

Feminist Critical Theory; Intersectionality; Democratic Deepening; Marked Bodies; Public Action.

\section{SUMARIO}

1. DESCENTRAR LAS POLÍTICAS PÚBLICAS CON UN ENFOQUE NO BINARIO. 2. LA PRAXIS INTERSECCIONAL EN EL DISEÑO DE POLÍTICAS PÚBLICAS. 3. LA METÁFORA Y EL JUEGO DE PALABRAS COMO HERRAMIENTA DE LA PRAXIS INTERSECCIONAL. 4. METÁFORAS E INTERSECCIONALIDAD EN EL CASO DE LA POLIITICA PÚBLICA DE PARTICIPACIÓN CIUDADANA. 5. CONCLUSIONES Y PISTAS DE INVESTIGACIÓN. REFERENCIAS BIBLIOGRÁFICAS.

\section{DESCENTRAR LAS POLÍTICAS PÚBLICAS CON UN ENFOQUE NO BINARIO1}

Aunque las definiciones sobre lo que es una política pública pueden ser más o menos restrictivas (Dye, 1972; Fontaine, 2015), todas coinciden en identificar una fuente pública de producción. Teóricamente, las políticas buscan poner soluciones a problemas y resolver demandas sociales. Ponen en el centro de su razón de ser la búsqueda de soluciones a las necesidades sociales de cada momento. Lo que está en disputa en el campo político es, justamente, la lectura que se hace del problema que hay que tratar de solucionar y, en base a eso, la imposición de una u otra decisión.

En la segunda mitad del siglo XX, con la naturalización y generalización del topos de la crisis de la democracia en los estados el Norte Global, los gobiernos ven la necesidad de modernizar las relaciones entre la ciudadanía y los agentes políticos en los procesos de toma de decisión. Asimismo, se asume la idea de que la acción pública debe de actualizarse y proyectar políticas para un sujeto «complejo». De modo que, junto al discurso de la crisis de la democracia y la legitimidad se integra el de la complejidad de las sociedades y de las necesidades que estas acogen (Martínez-Palacios, 2021).

Estas lecturas sobre la modernización orientan la acción pública a ajustar las soluciones a los problemas sociales que experimentan los individuos en lo cotidiano, y buscan minimizar el margen de error e ineficiencia política que tradicionalmente se ha asumido junto al discurso de la crisis de legitimidad. Así se explicaría que la forma burocrática de operar, basada en la especialización y la fragmentación, esté siendo revisada con otras como la nueva gestión pública.

Estamos ante un contexto ambivalente en el que conviven prácticas neoempresariales de gestión pública (privatización) y textos vehiculares de las políticas públicas en los que se busca centrar la comprensión de la eficiencia en términos de justicia social, y en los que se reconoce la diversidad que existe de experimentar los procesos vitales en función de la posición social de los individuos. Como muestra de lo anterior, tomando el caso español, es habitual encontrar preámbulos de textos jurídicos orientados a lograr una mayor justicia social que recogen las ideas de «reconocimiento de la complejidad de la sociedad» al tiempo que marcan una «voluntad de orientar la acción pública a objetivos de justicia social y sostenibilidad estructural» (cf. Ley $57 / 2003$, de 16 de diciembre de medidas para la modernización del gobierno local; Líneas generales de la reforma administrativa 2018-2020, Ministerio de Hacienda y Función Pública, entre otros productos jurídicos).

Ante la naturalización de la narrativa que sostienen estos textos, la teoría crítica feminista ha estudiado los límites y estrategias del «régimen de las apariencias», o del «cinismo político» que la recubre (Connell, 2005; Orozco, 2017; Fraser y Jaeggi, 2019; Martínez-Palacios, 2021). Se señala la contradicción de recoger por escrito los objetivos de una acción pública orientada a responder a la complejidad, poner en marcha prácticas administrativas que restringen los derechos de las personas e implementar una visión de la eficiencia productivista. Este trabajo de mantenimiento institucional a través de una política aparencial se ha hecho mediante muchos proyectos -la institucionalización de un gobierno de la performance (Bezez, 2020); la burocratización neoliberal (Hibou, 2012), o, más ampliamente, la naturalización de la ideología de Estado

1 Las autoras agradecen a las dos personas revisoras del manuscrito el tiempo y la energía empleada en este trabajo, así como la generosidad de las ideas aportadas y las referencias sugeridas. 
(Laval y Dardot, 2015; Boltanski y Chiapello, 2002)-. De entre todas las lógicas que recorren dichos proyectos de mantenimiento institucional a través de una política aparencial destacamos una, presente en las obras citadas hasta el momento y central para los trabajos de la teoría crítica feminista que motivan la presente reflexión. Se trata de la lógica de fragmentación que lleva de suyo un pensamiento binario.

La fragmentación de la práctica administrativa y el reconocimiento de la complejidad individual en los textos de ley tiene que ver con que la idea de lo complejo se conceptualiza tomando como medida para las políticas públicas la vida de lo que la economía feminista denomina sujeto BBVAh (Burgués, Blanco, Varón, Adulto/Asfaltado, heterosexual) con una funcionalidad y forma corporal normativa. Así, los ejercicios de abstracción necesarios en la elaboración de políticas públicas se basarían, según la crítica feminista, en diseñar políticas pensando en individuos con vidas fragmentadas en áreas (público vs. privado) y trabajos (productivo vs. reproductivo).

De igual modo, la teoría crítica feminista que orienta los trabajos de la presente reflexión se caracteriza por ofrecer herramientas para un diagnóstico del topos de la crisis, y destaca por su carácter propositivo. Así, desde la economía feminista se pone nombre al enfoque que recoge diversas propuestas prácticas que miran otramente las políticas, de forma no binaria, donde el centro no es la eficiencia en términos de acumulación, canalizada a través de la lógica de la fragmentación, sino que ponen su fin en una forma de justicia social que se concreta en mirar la acción pública con el enfoque en la sostenibilidad de la vida. No de cualquier vida, sino de una que merezca la pena ser vivida (Orozco, 2017).

Mirar la realidad desde la sostenibilidad de la vida «implica preguntarnos si al final, todo ese complejo engranaje (de elaboración de políticas públicas) permite a la gente que lo conforma comer o no, bien o mal, con soberanía alimentaria o sin ella, con tiempo de calidad para sentarse en una mesa, con compañía impuesta o elegida» (Orozco, 2017, p. 41). Significa interesarse por lo que parece indiscreto, y preguntar a las políticas públicas por temas insospechados por haber sido banalizados tal y como lo hacen los trabajos de investigación que movilizamos en los siguientes apartados de esta reflexión.

Asimismo, hacer desde el enfoque de la sostenibilidad de la vida pasa por construir desde lo Otro, hacer otramente, que se traduce en proyectar la acción pública desde posiciones de «outsider within» (Collins, 1986), «outsider incómodo y sensible» (Martínez-Palacios, 2021). Significa, hacer «partiendo de sí, para no quedarse en sí, para politizar la existencia y salir de sí» (Orozco, 2017, p. 41). Como proponemos en esta reflexión, esto requiere de otras herramientas políticas tales como la interseccionalidad y el pensamiento metafórico.

La teoría crítica feminista acordaría que quien ha tenido el poder de definir qué es una política pública hasta ahora, y en mayor medida en contextos de capitalismo financiarizado, no ha sido el outsider, sino el insider, y lo ha hecho hablando sobre vida, pero, pensando en una forma de vida fragmentada, asentada en la ruptura binaria matricial (productivo vs. reproductivo), encarnada en cuerpos infinitos que sostienen su trabajo público sobre otros que hacen el privado. Esta forma de ver la vida porta una visión binaria del mundo, contraria a sostener la vida de todas/os, que no asume una materialidad finita y vulnerable de los cuerpos, y el contexto que les rodea.

Por eso, a fin de romper con el binarismo que orienta la acción pública, la teoría feminista propone distintas herramientas que son comprendidas y explicadas como «marco epistemológico», "paradigma», «metodologías» o «enfoque», según con qué autora/autor pensemos, pero que se organizan, desde la década de 1989, siguiendo la metáfora que empleó la jurista feminista afroamericana Kimberle Crenshaw: la intersección.

La jurista empleaba un pensamiento alegórico para explicar la situación de las mujeres negras en los fallos de la jurisprudencia de los EEUU donde no se sabía si aplicar leyes de protección contra la discriminación racial, o leyes de protección contra discriminación de género (Crenshaw, 1989). De su reflexión se extrae una crítica a la idea de justicia que no reconoce, redistribuye, ni representa la vida de las personas que se ven atravesadas por múltiples ejes de dominación desde una posición subordinada.

Desde que Crenshaw bautizó como interseccionalidad la forma de vivir atravesada por distintos ejes de opresión han sido muchas las aportaciones al concepto. Para tratar de evitar el vaciado de contenido del término, hoy existe una literatura orientada a aclarar y poner en acción esta herramienta.

En ese contexto general de enmarque de las políticas y del momento que vive la literatura sobre la interseccionalidad y la acción pública, en las páginas que siguen, planteamos que las obras de Collins (2019), López y Platero (2019), y Marx y Pérez Bello (2018) invitan a aterrizar la praxis interseccional en la acción pública y, por lo tanto, sus aportaciones son interesantes para quienes diseñan e implementan políticas públicas.

Consideramos que al visibilizar y poner en marcha el pensamiento metafórico no binario cuya heurística ha sido estudiada por la metodología de la opresión (Sandoval, 2000), ayudan en la tarea de hacer políticas públicas interseccionales. En este artículo sugerimos que pensar y evaluar las políticas públicas desde 
figuras retóricas como las de Marx y Pérez-Bello (juegos de palabras) y metáforas como López y Platero (cf. «cuerpos marcados») llevan de suyo una mirada compleja de la realidad que hace que las preguntas a las políticas públicas sean radicalmente genuinas y no binarias tanto a la hora del diseño, como de su evaluación y proceso de puesta en marcha. Decimos esto porque esa forma de acceder a la acción pública no parte de una complejidad abstracta y retórica de la vida («la vida es compleja»), sino de la experimentación de formas de opresión derivadas de sistemas simplificados de organizar el mundo, cuyo mayor exponente es el pensamiento binario.

\section{LA PRAXIS INTERSECCIONAL EN EL DISEÑO DE POLÍTICAS PÚBLICAS}

A menudo, el auge de una temática o concepto en el campo académico se traduce en la proliferación de materiales dispuestos a explicar, definir e imponer significados al respecto. Las modas en la academia pasan por la híper-producción y tecnificación de una temática o concepto de modo que su sola mención se vuelva una herramienta para aumentar el capital cultural y académico del agente, y continuar con los ejercicios de distinción y división necesarios a la supervivencia de la lógica acumulativa del propio campo. Esta cuestión ya ha sido objeto de estudio por parte de distintas pensadoras (Collins y Bilge, 2016; Farinaz et al. 2016).

La preocupación por las tendencias a vaciar de significados esta herramienta nos deja trabajos como los de Patricia Hill Collins (2019) determinados por la idea de que: «sin una reflexión seria sobre sí misma, la interseccionalidad puede devenir simplemente otra teoría social que implícitamente mantenga el status quo». Sobre esta premisa, la autora explica en qué medida esta puede pensarse como teoría social crítica a partir de las escuelas de pensamiento de la teoría crítica de las escuelas de Frankfurt, de los estudios culturales británicos, o de la teoría social francófona de la década de 1950-60. La interseccionalidad aprendería de estas, y alcanzaría a esbozar las seis ideas centrales de su naturaleza crítica que rompen con la lógica de fragmentación y ruptura de todo binarismo: el contextualismo, la complejidad, la relacionalidad, las relaciones de poder, la desigualdad y la justicia social. El anclaje en ese conjunto de elementos haría de esta «una herramienta para resolver el problema de prácticas excluyentes de pensar solo en términos de género, raza o clase» (Collins, 2019, p. 107), es decir, el problema del binarismo en el proceso de producción de las políticas públicas.

La explicación de Collins sobre el término se desencadena de una práctica de poner en acción la solidaridad flexible entre las distintas entradas de la teoría crítica antes mencionadas, la teoría feminista, la teoría decolonial y la teoría crítica de la raza. Así, explica la autora que el terreno de la praxis, o la práctica reflexiva, crea un espacio más adecuado a los fines de justicia social y sostenibilidad de la vida que la teorización y conceptualización abstracta tradicional.

Esta praxis rompe con el binarismo de género, raza y clase, en tanto que lo nombra en cruce y de forma crítica. Así, se asienta contra toda forma dicotómica de pensamiento característica de la academia que viene de pensar que la teoría vale más que la práctica. De donde, luego, viene que el modelaje de las políticas públicas en abstracto y apoyada sobre axiomas, preceptos teóricos, o principios naturalizados (no discutidos, aceptados como dados, dóxicos) pero construidos socialmente, es la forma virtuosa de hacer lo público.

Precisamente, siguiendo la teoría feminista, esta forma se proyecta, de forma más o menos reflexiva, sobre cuerpos humanos y agentes sociales determinados. Agentes con formas determinadas que, en general, cumplen las normas y límites del pensamiento. Son cuerpos normativos, cuerpos BBVAh, «normales». Así, pensar en términos de interseccionalidad nos lleva a los cuerpos marcados por el cruce, y a interesarnos por las expresiones empleadas por estas personas a lo largo de la historia para explicar su posición. Nos lleva a una especie de democracia hermenéutica en la que para explicar la pobreza y empezar a diseñar políticas contra la pobreza no solo interese ir a los textos de los ilustrados sobre el asunto, sino que importe acudir a otra forma de materiales, dichos «profanos», que rompen con el logocentrismo (relatos de vida de personas indigentes, narrativas de personas sin hogar, estudio de la vida de los objetos que hacen lo cotidiano de las personas sintecho, entre otras).

Para ayudar a ese fin, en su último trabajo, Collins revisa algunos de sus planteamientos sobre las formas de relacionalidad interseccional entre los ejes que atraviesan las vidas de las personas. En sus obras anteriores estudiaba la adición de ejes, la asimetría en la articulación y co-formación de situaciones en las que se mezclan esas categorías como formas que conectan colateralmente con la interseccionalidad. Sin embargo, en este trabajo amplía la explicación y propone que las formas aditivas, la articulación, la coformación, todas, expresan alguna forma de relacionalidad interseccional que sirve a la teoría social crítica, y son útiles a la hora de ver de forma compleja un problema público que requiere de acción pública. 
Precisamente, la de la ontología de la opresión es una cuestión importante sobre la que parte de la literatura se ha centrado en discutir. Por eso, el desbloqueo que propone la autora al decir que lo aditivo, lo asimétrico, lo co-formado, lleva de suyo una relacionalidad interseccional, es importante para el estudio de las políticas públicas. Lo es porque su planteamiento facilita la comprensión y expresión de la experiencia vivida en términos de complejidad de la que dicen hablar las políticas en sus textos de ley. Asimismo, aclara la posibilidad de que la interseccionalidad sea una praxis a la que se puede acceder desde distintas puertas: la metáfora, la heurística o como palanca para un cambio de paradigma.

Siguiendo la lectura de Collins (2019), como heurística, esta herramienta estaría orientada a la solución de problemas sociales (pongamos la exclusión en políticas de participación) tratando de pensar el problema (la exclusión) con una lógica de cruce de ejes de opresión. Un ejemplo de ello lo encontramos en el proyecto «gure txutxu-mutxuak: Historias que hacen Zumarraga» (2019) cuyo objetivo es "crear un mosaico de miradas sobre la localidad a través de entrevistas y espacios de encuentro que ayuden a revalorizar el pasado, para (re)pensar el presente e imaginar el futuro en clave colectiva, en clave de cuidados comunitarios» (Aradia, 2021). En este caso se busca diseñar colectivamente políticas públicas comunitarias desde un pasado que ha sido excluido, viendo en la exclusión de historias y agentes que las encarnan una fuente para la producción de acción pública.

Asimismo, como palanca de paradigma tendría que ver con entender que las seis ideas centrales de la interseccionalidad son parte de una forma de ver el mundo que plantea nuevos problemas y nuevas soluciones al conocimiento. No se trata de decir que esta herramienta es en sí un paradigma nuevo, en los términos de Kuhn, para afrontar el diseño de políticas; se trata de entender que implica una forma de pensar otramente (pensar outsider) que conecta bien con algunos preceptos de la idea de paradigma del filósofo.

La interseccionalidad como metáfora es algo que atraviesa a ambas formas de verla anteriormente citadas (en el sentido en que cruce y palanca ya son, de hecho, metáforas). El pensamiento metafórico de Crenshaw (cruce, intersección) inaugura la etiqueta y la autora nos anima a pensar en la praxis interseccional orientada a las políticas para la justicia social, desde la heurística de la metáfora. Sobre esta cuestión consideramos que este ejercicio ya se está dando desde la teoría crítica feminista y que prueba de ello, así como ejemplo y modelo de lo que puede seguir haciéndose, nos lo dan dos obras que parten de una metáfora para ver lo complejo. Así, en esta reflexión, los trabajos de Silvia López y R. Lucas Platero (2019) y el de Daria Marx y Eva Pérez-Bello (2018), son entendidos como dos ejemplos de pensamiento metafórico y herramienta para la praxis interseccional en la acción pública.

\section{LA METÁFORA Y EL JUEGO DE PALABRAS COMO HERRAMIENTA DE LA PRAXIS INTERSECCIONAL EN LAS POLÍTICAS PÚBLICAS}

Siendo hacer políticas públicas no binarias orientadas a la sostenibilidad y democratización de la vida que merezca ser vivida un objetivo, los dos trabajos arriba citados proyectan acción pública a través de una metáfora relacionada con el cuerpo.

Un cuerpo puede estar marcado de muchas formas. Esto es porque casi todo en él habla. Habla su forma. Por ejemplo, un cuerpo gordo vive sabiendo que proyecta la gordura, y que esta significa todo lo que Marx y Pérez-Bello señalan: «falta de fortaleza mental, vulnerabilidad en salud, falta de determinación» (2018, pp. 19-23). Lo que nos interesa subrayar aquí es que estas proyecciones convertidas en estigmas son arrastradas en el proceso de elaboración de políticas públicas. Por eso, algunos cuerpos aprenden que tienen que esconderse porque están marcados (López y Platero, 2019). Con esta metáfora sobre los cuerpos marcados buscan «contribuir a explorar un debate aún poco frecuente en el análisis de políticas públicas producido en el contexto español: un tipo de evaluación de la acción política que ponga en el centro del análisis el cuerpo, sus bienes y su sufrimiento» (López y Platero, 2019, p. 13). En un contexto de "emergencia del cuerpo» (Turnes, 1994 en Esteban, 2013, p. 27) en el que se da «la importancia de ciertos elementos directamente relacionados con esa cultura del cuerpo, el consumismo y su crítica, el poder creciente de la autoridad médica y la crítica feminista» (Esteban, 2013, p. 27), hablar sobre el cuerpo, con el cuerpo, en las políticas públicas es pertinente. La cuestión es cómo hacerlo.

La metáfora de cuerpo marcado es una herramienta sugerente para pensar en el cómo porque introduce un pensamiento interseccional, explícitamente no binario, en el análisis de las políticas públicas. La hipótesis que se encuentra en el origen de la metáfora es explícitamente antibinarista ya que parte de la idea de que «la acción política distribuye diferencialmente entre la población condiciones vitales como el acceso al 
sistema de salud, educación, etc. (...) la acción política se concreta en procedimientos administrativos que pueden tener la capacidad de violentarnos o humillarnos al refinar categorías como el binarismo de género» (López y Platero, 2019, p. 14).

Así, las/os autoras/es dan forma al cuerpo marcado como expresión de praxis interseccional para estudiar las políticas públicas. Esos cuerpos marcados son, por naturaleza: vulnerables, finitos e inter-ecodependientes. Son cuerpos abyectos y disidentes: cuerpos desordenados (Burgos, 2019, pp. 27-50, en López y Platero, 2019). Quienes desordenan, los agentes outsider, ven hacia qué cuerpos se orientan las políticas públicas y en qué sentidos concretos son excluyentes. Lo ven porque lo padecen y resisten, son vulnerables ante la norma y resistentes hacia sus efectos. Siguiendo este planteamiento, la vulnerabilidad de los cuerpos, su reconocimiento, nos permitiría, por ejemplo, al diseñar una política, al implementarla y al evaluarla preguntarnos cuestiones cómo: ¿A quién se imagina como vulnerable el agente decisor en esas políticas, no binarias, orientadas a una vida que merezca ser vivida? ¿Qué cuerpo imagina? ¿Cómo marca esa política posteriormente ese cuerpo? ¿Cómo se resiste ese cuerpo?

Con una misma centralidad en el cuerpo marcado, pero usando una expresión distinta, en este caso, una que viene de un juego de palabras que tiene sentido en francés (gros mot, insulto) Daria Marx y Eva Pérez-Bello (2018) ofrecen un ejemplo encarnado con el que reflexionar sobre los límites de las políticas sanitarias, educativas, mediáticas, reproductivas, de salud mental o culturales desde un cuerpo marcado gordo. La gordofobia les sirve a las autoras para explicar la forma en la que la diversidad funcional, el género, la edad, la clase social, la raza, intersecta en unos cuerpos que se ven obesos y no entran, literalmente, en los instrumentos que necesitan las políticas para llevarse a cabo. Esto pasa, por ejemplo, porque los aparatos sanitarios del estado no estén preparados para pesar a personas de más de 120 kilos, ni para tomarles la tensión, ni para ofrecerles una pastilla anticonceptiva que responda a las necesidades (cf. caso de la pastilla anticonceptiva Norlevo que no tiene eficacia a partir de 70 kilos).

Así, desde una visión crítica con el pensamiento binario «gordo=malo vs. flaco=bueno», hacen la crónica de una discriminación cotidiana y evalúan, sin protocolo ni plantilla estandarizada más que la que les da las marcas del cuerpo, las políticas públicas francesas. Eso les permite acceder a una heurística interseccional e indagar, por ejemplo, en el olor como herramienta que usa el estigma a la hora de elaborar políticas públicas. Exponen cómo los cuerpos marcados remiten a olores, con efectos de muerte al estilo del filme Parásitos (Bong Joon-ho, 2019). El estigma construido sobre la falsa idea de que el cuerpo gordo que huele a sudor por la grasa excluye físicamente a las personas del espacio público libre. La crónica diaria de los cuerpos marcados gordos pone de manifiesto que una política pública no binaria que ponga en el centro la vida tiene que ser altamente sensible a lo encarnado, tanto objetivado, como incorporado. Ésta partiría de outsiders incómodos y altamente sensibles que buscan democratizar su posición de vigilantes de las prácticas injustas de los Estados.

Ambos trabajos, centrados en no hacer políticas públicas binarias a la luz de la heurística de la metáfora del cuerpo, nos recuerdan que «el estudio del cuerpo como objeto central de las Ciencias Sociales (...) es un enfoque relativamente nuevo (...)» y muy sugerente, como se ve del trabajo de las autoras de las obras seleccionadas, ello «nos debería de llevar a matizar más los análisis concretos sobre el cuerpo y a prestar más atención a los cambios históricos y a la variabilidad interna de cada grupo social» (Esteban, 2013, pp. 23-31).

\section{METÁFORAS E INTERSECCIONALIDAD EN EL CASO DE LA POLÍTICA PÚBLICA DE PARTICIPACIÓN CIUDADANA}

Muchas de las propuestas de la teoría crítica feminista que se centran en orientar las políticas públicas hacia lugares no binarios en los que la vida que merezca ser vivida sea puesta en el centro tienen en común dos cosas. Por una parte, el pensamiento metafórico que introduce alguna relacionalidad interseccional entre los distintos ejes que estructuran la vida de las personas. Por otra, comparten un sentido práctico orientado a la acción transformadora en la que la dimensión participativa y/o deliberativa del procedimiento de llegar a las políticas públicas sostenibles tiene una importancia central. Es decir, la teoría crítica feminista invita a que la praxis descanse en la toma de decisiones no binaria colaborativa, y comunitaria en la que se introduzcan formas de hacer participativas, deliberativas o del desarrollo comunitario.

Este acuerdo cada vez más generalizado sobre la fe participativa nos conduce a explorar con la herramienta de la interseccionalidad y la metáfora las políticas públicas de participación ciudadana. Estas son, a la vez, una línea sectorial de políticas, y una herramienta para canalizar la estrategia de toma de decisión participada. 
El optimismo de la teoría crítica feminista con relación a los diseños colaborativos para reflexionar conjuntamente puede ser mirado con recelo si consideramos la literatura crítica de la deliberación y la participación ciudadana (Martínez-Palacios, 2021). Esta literatura indica que las políticas participativas también usan las marcas normativas de los cuerpos, del modo en el que lo hemos estudiado hasta ahora en este texto. Pero, además, añaden una forma más «sofisticada» de exclusión porque recoge aspectos incorporados del estigma que cobran una importancia central en espacios en los que la retórica y el diálogo canalizan el debate. Con esto subrayamos la importancia de tomar en consideración la dimensión metapolítica de las políticas públicas que buscan no reproducir el pensamiento binario y ponen la vida en el centro.

Conviene recordar que el pensamiento metafórico ha sido también prolífico en la literatura de las políticas de la participación, «la escalera de la participación» de Arnstein (1969); «los desbordes creativos» del ilusionismo social (Villasante, 2017), «la rueda de la participación» (Davidson, 1998) son algunas muestras que lo ejemplifican.

Para la problemática que tratamos en esta reflexión nos interesa rescatar la metáfora de la escalera de Arnstein porque conecta con el cinismo político, o la política de las apariencias, a la que se hacía referencia en la introducción de este artículo. Según esta escalera existirían ocho peldaños que en la acción pública se caracterizan como participación y que, sin embargo, esconden prácticas de manipulación no participativas (cf. imagen 1). La autora identificaba, en 1969, las formas de participación aparencial que instituye la administración pública en nombre de la toma de decisión participada. Manipulación, terapia, información, consulta, apaciguamiento, asociación, delegación de poder y control de poder son los ocho peldaños que nos propone Arnstein. Esta indica que los seis primeros peldaños, aunque sean denominados «participativos», tratan de apaciguar y manipular a la ciudadanía a través de consultas no vinculantes, tomas de decisión en las que la presencia de la ciudadanía es simbólica, no por el número de participantes, sino porque al no conocer los códigos del debate esta no puede introducir propuestas realmente considerables. Esto tendría que ver con ese tipo de exclusión que se da cuando lo que está en juego no se desvela y los códigos de acceso a los espacios no aparecen escritos en ningún lado (códigos de conducta, formas lingüísticas, procedimientos no escritos, entre otros) y sin cuyo conocimiento nada de lo que se diga tendrá validez. En definitiva, lo que esta escalera pone de manifiesto es la existencia de una lógica binaria basada en el dualismo «docto vs. profano», en la que lo primero se asigna a los agentes técnicos y políticos y lo segundo a la ciudadanía.

IMAGEN 1. EscaleRA DE LA PARTICIPACIÓN DE ARNSTEIN

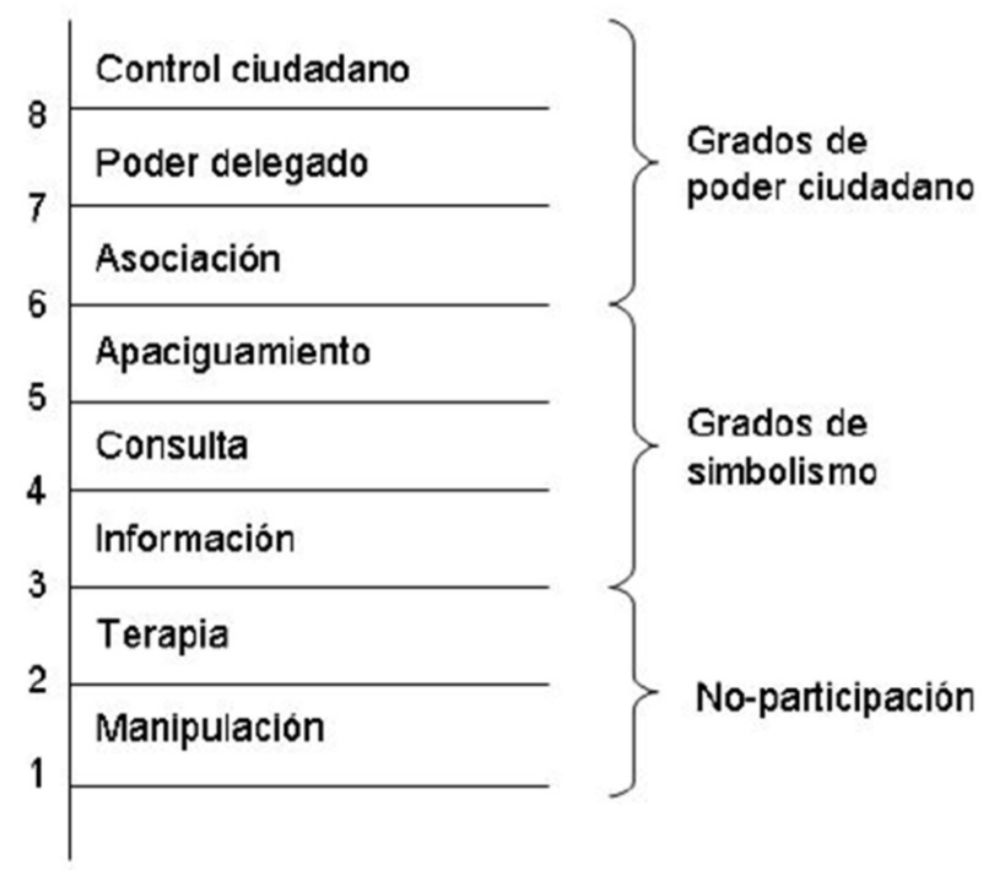

Fuente: Arnstein, 1969, p. 217. 
De los trabajos de Arnstein y de la literatura crítica de la participación, sabemos que los procesos de decisión participados y colaborativos son escasos dentro de la acción pública y que cuando se dan, además, se ven atravesados por las estructuras de género, de raza, de edad, de clase social, de diversidad funcional y corporalidad, entre otras. En ese sentido, cabe destacar, para el objetivo del texto -pensar en políticas que desactiven un pensamiento binario que aleja al objetivo de una vida que merezca ser vivida, todo, con la herramienta de la interseccionalidad-, que el camino para hacerlo (la participación) está, también, sesgado porque es un instrumento de marcación de los cuerpos (Chevallier, 2019).

Así, considerando la escalera de Arnstein y centrándonos ya solo en lo que puede ser considerado grado de poder ciudadano, sabemos que los espacios de deliberación y participación priorizan las formas discursivas de los BBVAh con cuerpos normativos y funcionales a una forma de entender la eficiencia vinculada a la acumulación de resultados rápidos (Mansbridge, 1990; Young, 2000; Fung y Wright, 2003; Warren, 2009; Martínez-Palacios, 2021). En esa forma de participación se priorizan discursos desapasionados y formas de intervención que se enfundan en cuerpos marcados «normales» que desprenden hexis corporales «normales». En este sentido la metáfora de cuerpos marcados abre otras vías al pensamiento interseccional al permitir entender que estos remiten a una dimensión incorporada en sus movimientos y que, aunque una consiga un cuerpo en apariencia «normal» y aceptable según el canon BBVAh, si los gestos no acompañan éste queda expulsado sin necesidad de una norma que indique su exclusión. Esas marcas más «inmateriales» tienen que ver con el habitus (Bourdieu, 1972) y los elementos que lo construyen: el acento, el movimiento, el saber sentarse, el saber el protocolo, el sentido práctico; son marcas que vienen de normas que el derecho no regula pero que funcionan en la práctica de la acción pública y que ayudan a marcar los cuerpos.

Un ejemplo de todo esto en políticas públicas de participación podría ser el siguiente: siguiendo la metáfora de Arnstein, un proceso de presupuestación participada en materia de sanidad que integre a personas con cuerpos marcados gordos, con diversidad funcional, marcados por el género la edad, el origen y la sexualidad dominantes y por ello, excluidos de las formas hegemónicas de pensar la salud primaria, podría verse como un primer intento de reflexionar una política que pone en cuestión el modelo de sociedad completo.

El diseño reflexivo con los cuerpos marcados, y canalizado de forma colaborativa, pasaría por interrogar no solo las marcas del cuerpo (que pueden estar presentes en forma física), sino las marcas de las que el derecho no habla: debería de ser reflexivo en cada contexto con el hecho de que en la cultura occidental el tipo de argumento que domina el espacio público es el racional, donde esto significa discurso justificado en base a criterios considerados de bien general e interés público, donde lo experiencial no cuenta.

Asimismo, debería de ser reflexivo con el hecho de que cuando unos cuerpos hablan y lo hacen con un acento sus discursos cobran más valor que otros (catalán, francés frente al latino, andaluz). Es decir, sería reflexivo sobre que el binarismo no solo pasa por los cuerpos marcados que ya llevan una dimensión impresa e incorporada en el cuerpo, sino que la expresión del cuerpo también se ve influida por el valor de lo que este transmite.

Esa reflexión sobre la participación lleva una carga auto-reflexiva de los agentes que diseñan para quienes el pensamiento metafórico y la interseccionalidad puede ser de gran utilidad siempre que su objetivo sea trabajar de forma no binaria para no generar vidas excluidas. Esta carga auto-reflexiva ha mostrado ser central en las experiencias prácticas de políticas públicas diseñadas desde un enfoque interseccional y participativo que no refuerzan una visión binaria de la acción pública (Martínez-Palacios, 2017). Existen casos como los del proyecto de la «Atalaya de la Soledad» del Ayto. de Zumarraga, orientado a crear el I Plan de Acción para la detención de la soledad no deseada entre personas mayores (2020) que trabaja una temática que remite a la exclusión (soledad), de forma participada e interseccional (Aradia, 2021). Lo que propone este texto es que para profundizar en esa tendencia y seguir experimentando con ella, la creatividad metafórica puede ser una herramienta de gran ayuda.

\section{CONCLUSIONES Y PISTAS DE INVESTIGACIÓN}

Esta reflexión parte de trabajos que muestran que son muchas las formas de marcar el cuerpo y que las políticas públicas hoy no son inherentes a emplearlas, de manera más o menos reflexiva, para excluir a quienes viven las marcas. Una acción pública no binaria que ponga la vida en el centro no puede abstraerse de tal realidad y por eso interesan los instrumentos para una práctica administrativa diferente que no se base en la lógica de la fragmentación y la ruptura y que, ante contextos de alta complejidad y crisis, propongan medidas de alta sensibilidad. 
En este texto hemos expuesto que los cuerpos marcados dan sentido práctico a la propuesta de Collins (2019) de pensar la interseccionalidad en metáfora. Asimismo, esta metáfora permite pensar en políticas no binarias que ponen la vida en el centro y amplían su heurística porque estudiando las marcas del cuerpo, permiten acceder a la dimensión incorporada de la vida. Abren las puertas a pensar en una política de los sentidos (una política de alta sensibilidad), sin por ello despegarse de las estructuras materiales que la sostienen.

En esta reflexión sobre la importancia de diseñar políticas de alta sensibilidad, y siguiendo a Crenshaw cuando propuso la noción de interseccionalidad para la gestión jurídica, «no es necesario creer que el consenso político para poner el foco en las vidas de las personas que tienen una intersección atravesada por infinitud de estructuras vendrá mañana. Es suficiente por hoy saber que se hace un esfuerzo por saber que esas posiciones existen y que todo lo que no incluya a esas personas será autocomplaciente» (Crenshaw, 1989, p. 166). Mañana es hoy, y hoy no se trata de hacer políticas de los sentidos, sin embargo, algo habrá que haber avanzado en 20 años; tal vez no en términos de consenso, pero sí en términos de tener herramientas para identificar dónde falla lo que se presenta como justo.

Así, hoy en el paradigma neoliberal en la gestión de las políticas públicas a raíz del contexto surgido con la crisis de la COVID-19, no buscaríamos responder a la política de las apariencias con política estética. No obstante, parece urgente emplear un pensamiento interseccional que recoja la complejidad con la que se experimenta la exclusión y con la que se resiste, tal y como lo muestran las últimas investigaciones sobre políticas públicas en tiempos de Covid (van Barneveld, et al., 2020; Hankivsky, et al. 2021). Esta tarea puede hacerse a través de metáforas que organicen las evaluaciones de políticas públicas, y que reconozcan que esos recursos figurativos son ricos hasta el punto de permitir una reflexión sobre el olor, o el ruido, para identificar qué estructuras de clase, de raza, entre otras, afectan en una sociedad.

Esta cuestión es importante porque el impacto de un modelo de sociedad sobre el comportamiento de las personas se refleja en las mismas políticas públicas desde su elaboración hasta su evaluación, pasando por su implementación. Así, importaría saber que organizar los espacios asépticos, calmados, serenos, limpios, neutros olfativamente hablando, que son los que se priorizan en las salas de tomas de decisión, marcan a quienes entran, no solo con la estética a la que remite el cuerpo; les marca en lo más íntimo haciéndoles entender que aquel no es su lugar.

\section{REFERENCIAS BIBLIOGRÁFICAS}

ARADIA (2021). Espacio para la profundización democrática. Proyectos. Recuperado el 28 de octubre de 2021 de https://www.aradiacooperativa.org/acompañamiento/

Arnstein, S. (1969). A ladder of citizen participation. Journal of the American Institute of Planners, 35(4), 216-224. https://doi.org/10.1080/01944366908977225

Bezez, P. (2020). Le nouveau phénomene bureaucratique. Le gouvernement par la performance: entre bureaucratisation, marché et politique. Revue française de Science politique, 70, 21-47. https://doi.org/10.3917/ rfsp.701.0021

Boltanski, L. y Chiapello, E. (2002). El nuevo espíritu del capitalismo. Akal.

Bourdieu, P. (1972). Esquisse d'une théorie de la pratique. Droz.

Chevallier, T. (2019). Résister à bas bruit aux catégorisations institutionnelles dans des dispositifs de participation à Berlin. Participations, 25, 109-138. https://doi.org/10.3917/parti.025.0109

Collins, P. H. (2019). Intersectionality as critical social theory. Duke University Press.

Collins, P. H. (1990). Black Feminist Thought. Knowledge, consciousness, and the politics of empowerment. Routledge.

Collins, P. H. (1986). Learning from the ousider witin. The sociological significance of Black Feminist thought. Sociological Problems, 33(6), 14-32. https://doi.org/10.2307/800672

Collins, P. H. y Bilge, S. (2016). Intersectionality. Polity Press.

Connell, R. (2005). Advancing Gender Reform in Large-scale Organisations: A New Approach for Practitioners and Researchers. Policy and Society, 24(4), 5-24. https://doi.org/10.1016/S1449-4035(05)70066-7

Crenshaw, K. (1989). Demarginalizing the Intersection of Race and Sex: A Black Feminist Critique of Antidiscrimination Doctrine, Feminist Theory and Antiracist Politics. University of Chicago Legal Forum, 1989, article 8. https:// chicagounbound.uchicago.edu/uclf/vol1989/iss1/8

Davidson, S. (1998). Spinning the wheel. Community planning, 1, 14-15. Recuperado el 28 de octubre de 2021 de https://sarkissian.com.au/wp-content/uploads/sites/13/2009/06/Davidson-Spinning-wheel-article1998.pdf

Dye, T. R. (1972). Policy Analysis And Political Science: Some Problems At The Interface. Policy Studies Journal, 1, 103-107. https://doi.org/10.1111/j.1541-0072.1972.tb00077.x 
Esteban, M. L. (2013). Antropología del cuerpo. Bellaterra.

Farinaz, F., Lépinard, E. y Roca i Escoda, M. (2016). L'intersectionalité: enjeux théoriques et politiques. La Dispute.

Fontaine, G. (2015). El análisis de políticas públicas. Conceptos, teorías y métodos. Anthropos-FLACSO.

Fraser, N. y Jaeggi, R. (2019). Capitalismo. Una controversia desde la Teoría Crítica. Morata.

Fung, A. y Wright, E. (2003). Deepening Democracy: Institutional innovations in empowered participatory governance. Verso.

Hankivsky, O. y Kapilashrami, A. (2021). Beyond sex and gender analysis: an intersectional view of the COVID-19 pandemic outbreak and response [Policy Brief]. Global Policy Institute, Quen Mary University of London. https:// www.qmul.ac.uk/gpi/media/global-policy-institute/Policy-brief-COVID-19-and-intersectionality.pdf

Hibou, B. (2012). La bureacratisation du monde à' l'ére néoliberale. La découvérte.

Joon-ho, B. (dir.)(2019). Parásitos [película]. Barunson E\&A.

Laval, C. y Dardot, P. (2015). La nueva razón del mundo. Gedisa.

López, S. y Platero, R. L. (2019). Cuerpos marcados. Vidas que cuentan y políticas públicas. Bellaterra.

Mansbridge, J. (1990). Beyond Adversary Democracy. The University of Chicago Press.

Martínez-Palacios, J. (2021). El giro participativo neoliberal. UPV/EHU.

Martínez-Palacios, J. (2017). Innovaciones democráticas feministas. Dykinson.

Marx, D. y Pérez-Bello, E. (2018). Gros, n'est pas un gros mot. Chroniques d'une discrimination ordinaire. Librio.

Orozco, A. P. (2017). Subversión feminista de la economía. Aportes para un debate sobre el conflicto capital-vida. Traficantes de Sueños. https://www.traficantes.net/libros/subversi\%C3\%B3n-feminista-de-la-econom\%C3\%ADa

Sandoval, C. (2000). Metodology of the Oppressed. Universidad de Minesota.

Van Barneveld, K., Quinlan, M., Kriesler, P., Junor, A., Baum, F., Chowdhury, A., Junankar, P. (Raja), Clibborn, S., Flanagan, F., Wright, C. F., Friel, S., Halevi, J., y Rainnie, A. (2020). The COVID-19 pandemic: Lessons on building more equal and sustainable societies. The Economic and Labour Relations Review, 31(2), 133-157. https://doi. org/10.1177/1035304620927107

Villasante, T. (2017). Democracias transformadoras. El viejo topo.

Warren, M. (2009). Governance-driven democratization. Critical Policy Studies, 3, 3-13. https://doi. org/10.1080/19460170903158040

Young, I. M. (2000). Inclusion and Democracy. Oxford University Press. 